\title{
LA CATEDRAL DE SANTA ANA DE LAS PALMAS: DIMENSIÓN URBANA Y TERRITORIAL
}

\author{
Juan Sebastián López García
}

\section{RESUMEN}

El mapa episcopal canario es resultado de un proceso de varias centurias, a partir del siglo XIV. La consolidación definitiva del obispado se produjo con el traslado efectivo de la sede desde Rubicón (sur de Lanzarote) hasta Gran Canaria tras su conquista en 1483. A partir de ese momento tres edificios sucesivos han sido sede de la catedral canariense, ocupando siempre un espacio urbano central en la ciudad, desde la plaza de San Antón, en el núcleo fundacional, hasta la definitiva plaza de Santa Ana, donde actualmente preside el espacio público principal de Las Palmas de Gran Canaria. La construcción de la catedral de Santa Ana se convirtió en la iniciativa arquitectónica más importante de Canarias desde principios del siglo XVI con una importante ampliación en el siglo XVIII y reformas durante el siglo XIX, especialmente en la fachada. Por su importancia influyó en otros edificios del territorio del archipiélago.

Palabras Clave: catedral, obispados, plazas, ciudad episcopal.

ST. ANNE'S CATHEDRAL IN LAS PALMAS:

URBAN AND TERRITORIAL DIMENSION

Abstract

The diocesan map of the Canary Islands is the result of a long process which lasts several centuries after it starts in the Fifteenth Century. The consolidation of the bishopric took place with the transfer of its seat from the Rubicon (south of Lanzarote) to Gran Canaria after its conquest in 1485 . From that moment onwards three succesive buildings have been the seat of the canariensis Cathedral. They have always occupied central urban spaces of the town, starting with the "Plaza de San Antón" in the foundational nucleus, until its final location in the "Plaza de Santa Ana", where it presides to this day over the main public space of Las Palmas de Gran Canaria. The construction of the catedral became the most important architectural undertaking in the Canary Islands from the start of the Fifteenth Century, undergoing an important extension during the Eighteenth Century, and several further changes during the Nineteenth, particularly regarding its facade. It did exert its influence over other buildings of the canarian territory, specially during the Eighteenth Century.

KEYWORDs: cathedral, bishoprics, squares, episcopal city. 


\section{INTRODUCCIÓN}

«... eso dura más que la fábrica de Santa Ana», es el comentario que aún se puede oír por algún rincón de Gran Canaria cuando una obra tarda más de lo normal en ejecutarse. El dicho popular ya indica un par de características de la catedral de Las Palmas, la primera es que los grancanarios en un sentido muy familiar la llaman por el nombre propio y la otra es que en el imaginario colectivo la construcción no se acaba nunca, lo que la vincula a muchas generaciones, muchos períodos históricos y momentos artísticos diferentes.

La santa iglesia catedral basílica de Santa Ana de Canarias, o la catedral de Las Palmas, es la sede episcopal de la diócesis canariense y rubicense, o lo que es lo mismo, del obispado de las islas orientales. Por su valor histórico y artístico es el monumento más importante de la comunidad autónoma, declarado Bien de Interés Cultural en $1974^{1}$.

\section{DIÓCESIS EN UN TERRITORIO INESTABLE}

El mapa episcopal canario comenzó a perfilarse en el siglo XIV y se podría decir que aún no está concluido a falta de constituir una provincia eclesiástica propia. Las diferentes diócesis que han existido y existen son expresión de etapas de la historia del archipiélago, y la territorialidad eclesiástica de distinto alcance, desde la jurisdicción del obispado hasta los límites de las parroquias, explica en la mayoría de los casos la situación de determinados momentos históricos de más de seis siglos. En el ámbito oceánico de nuestro entorno «La primera gran plataforma misionera la constituyeron las islas Canarias. Se trata de un descubrimiento atlántico que conmovió la Europa cristiana del siglo xiv. Descubiertas por los mercaderes italianos, llega su noticia a la misma corte pontificia en donde se promueven reiteradamente planes para vincularlas definitivamente a la Cristiandad». En ese entonces estarán presentes dos características que luego se verán en América: «La primera, la vinculación a los conquistadores y gobernantes de las islas Canarias y mediante de ellos a la Corona de Castilla. La segunda, la relación de dependencia estricta en que se encontrará respecto a los frailes misioneros, en este caso los franciscanos de Castilla ${ }^{2}$.

Los descubrimientos geográficos del siglo xIV y el inicio de un interés real por estas tierras y sus compañeras se manifiesta de forma clara en la creación del obispado de la Fortuna o diócesis de Telde en 1351, por bula de Clemente VII de 7 de noviembre 3 . Esta circunscripción misional, in partibus infidelium, en tierra no conquistada, va unida a la acción evangelizadora de frailes mallorquines y su

${ }^{1}$ D. 1689/1974, de 24 de mayo de 1974. BOE 148, 21.06.1974.

2 García Oro, José y Portela Silva, María José: La Iglesia de Canarias en el Renacimiento: de la misión a la diócesis. Estudio histórico y colección diplomática, Puerto del Rosario, 2005, p. 43.

${ }_{3}$ Rumeu de Armas, Antonio, El Obispado de Telde. Misioneros mallorquines y catalanes en el Atlántico, Madrid-Las Palmas, 1960. 
temprana presencia en las islas, en un territorio que no se consigue integrar en ese momento, aunque quedarán huellas de su presencia.

El tanteo territorial del XIV es un importante antecedente para el Xv, siglo que supone el paso decisivo para la consolidación del proceso episcopal en el archipiélago, de tal manera que la situación actual es herencia directa de esa centuria. La creación por Benedicto XIII en virtud de la bula de 7 de julio de 1404 del obispado del Rubicón, en el sur de Lanzarote, suponía la posibilidad de una sede con obispo residente en una isla cristianizada, especialmente porque la bula incluía aspectos relacionados con el urbanismo y la arquitectura, ya que el lugar llevaría el título de ciudad y el rango de catedral para el edificio, aunque en la práctica no fuera así. En ese período de la conquista iniciado por Bethencourt en 1402 sólo se integraron en la corona de Castilla cuatro de las islas (Fuerteventura, La Gomera, El Hierro y Lanzarote), con un sistema administrativo de carácter señorial y con escaso desarrollo urbano, donde sólo destacaban las incipientes cabeceras insulares y con diferencias según cada una ${ }^{5}$. Dos décadas después de la erección episcopal del Rubicón se crea un obispado exclusivo para Fuerteventura, el tercero que existió en Canarias, que permaneció durante un corto período de tiempo en el que el archipiélago tuvo dos diócesis (1424-1433) ${ }^{6}$. La consolidación de la presencia portuguesa en la mayoría del ámbito macaronésico y la nueva dirección geopolítica de esta zona del Atlántico en las rutas africana y americana en el contexto de 1492 aumentan el interés de la corona castelllana por completar la conquista de Canarias (1478-1496), con la incorporación de las islas de realengo (Gran Canaria, La Palma y Tenerife). La toma

${ }^{4}$ El texto de la citada bula dice: «... Es conocido como la isla de Lanzarote y otras cercanas de Canarias fueron adquiridas por ciertos cristianos y a los nativos se les ha predicado la fe cristiana que han recibido en gran parte, mientras que se espera que otros igualmente la recibirán. Por lo cual nosotros, queriendo promover la Fe Católica y sabedores que en dicha isla está el fortín de Rubicón con la iglesia de San Marcial, determinamos nombrarla ciudad episcopal, constituir en catedral e iglesia episcopal a la citada iglesia y constituirla sede episcopal para esta isla y para las islas vecinas. Esta iglesia será sufragánea de la de la iglesia metropolitana de Sevilla...», (Vid. García Oro y PorTEla SiLva, op. cit., p. 123.); «... Sabemos que en el Castillo del Rubicón de la Isla de Lanzarote, se ha edificado una Iglesia bajo la advocación de San Marcial [...] y queriendo distinguir aquel Castillo y aquella Iglesia con algún favor Apostólico, después de una madura deliberación de nuestros hermanos, por consejo de ellos y de la plenitud de nuestra autoridad apostólica, para loor del nombre de Dios, gloria y exaltación de su Santa Iglesia, dilatación de la fe y mayor utilidad de las almas, erigimos el referido Castillo del Rubicón, supuesto que tiene proporción para ello, en ciudad, y la honramos con el nombre de tal, siendo nuestra voluntad que se llame Ciudad Rubicense; y señalamos por su Diócesis el resto de su Isla y todas las otras comarcanas. Y la dicha Iglesia, de consejo de los dichos nuestros hermanos, la hacemos y establecemos Catedral, y la condecoramos con el título de la Dignidad Episcopal...» (Vid. SÁnchez Rodríguez, Julio: La Iglesia en las Islas Canarias, Las Palmas de Gran Canaria, 2004, pp. 34 y 35.

5 López García, Juan Sebastián: "Canarias: hacia un sistema urbano, siglos XV y XVI», Ciudad y Territorio, n. ${ }^{\circ} 77-3$, (1988), p. 4.

${ }^{6}$ Cazorla León, Santiago y Sánchez Rodríguez, Julio, Obispos de Canarias y Rubicón, Madrid, 1997, pp. 26 y 27. Sánchez Rodríguez, op. cit., p. 153. Wölfel, Dominik Josef: «El efímero obispado de Fuerteventura y su único obispo», Investigación y progreso, marzo (1934). García Oro y Portelo Silva, op. cit., pp. 48-49. 
de posesión de Gran Canaria en 1483 supone tener la primera isla administrada directamente por la corona, en cuyo proceso destacará la presencia de Juan de Frías, obispo titular de Rubicón, clave para la nueva ordenación territorial del archipiélago desde la institución eclesiástica, pasando de una situación inestable a otra organizada.

\section{UNA SEDE EPISCOPAL TRASLADADA: DE RUBICÓN A LAS PALMAS}

Juan de Frías es un personaje muy destacado, ya que su presencia en la conquista de Gran Canaria y su condición de obispo de Rubicón fueron posiblemente determinantes para el arranque de la que sería la ciudad de Las Palmas?. Él era la única autoridad reconocida con alcance jurisdiccional a todas las islas, que aunque fuera eclesiástica no era nada desdeñable para la época. En realidad no tenía sede episcopal física, dado que Rubicón era un desértico paraje del sur de Lanzarote y la cabecera de esa isla se había consolidado en la villa de Teguise ${ }^{8}$.

Con la finalización de la guerra de Canaria se produjo la conversión del campamento militar, desde donde se organizó la conquista de la isla, en un núcleo poblacional. Las Palmas se constituyó en la nueva capital insular y en la primera ciudad de realengo del archipiélago, ya que las demás villas estaban en territorio de señorío. Se debe reconocer la importancia que tuvo el obispo Frías, como autor de una estrategia cuyo resultado y consecuencia fue clave para consolidar un obispado que poco futuro tenía entonces en su sede oficial de Lanzarote y que no había conseguido organizarse en casi ochenta años. La decisión del traslado se había determinado décadas atrás cuando el obispo Calvetos lo solicita a Eugenio IV, papa que lo autoriza el 25 de agosto de 1435. Esta circunstancia no se hizo efectiva y hay otra segunda autorización del papa Sixto IV de 29 de agosto de $1492^{9}$. El traslado beneficiaba a varios agentes, pero sobre todo a la propia institución porque iba a contar con los mecanismos necesarios a la hora de organizarse ex novo y dotarse de los dos elementos esenciales de los que careció hasta entonces: sede catedralicia y mansión del prelado. Al mismo tiempo, para su mantenimiento el obispado recibió la jurisdicción del señorío de Agüimes ${ }^{10}$.

Hay que considerar que para Las Palmas, urbe recién fundada, la condición episcopal constituyó el pilar más importante, para su evolución y rápida consolidación

7 Cazorla León y Sánchez Rodríguez: op. cit., pp. 47-53. García Oro y Portelo SILVA, op. cit., pp. 74-78.

${ }^{8}$ López García, Juan Sebastián: Origen y desarrollo urbano de Teguise (Lanzarote), Las Palmas de Gran Canaria, 1993, pp. 1-2.

9 Cazorla león y Sánchez Rodríguez: op. cit., p. 30. Cazorla León, Santiago: Historia de la Catedral de Canarias, Las Palmas de Gran Canaria, 1992, pp. 5-9. SÁnchez Rodríguez: op. cit., pp. 53-55.

10 Vid. Cazorla León, Santiago: Agüimes, Real Señorío de los Obispos de Canarias (14861837), Las Palmas de Gran Canaria, 1984. 
en el territorio canario, ya que a falta de otras instituciones civiles de ese rango, de alguna manera la convertía en cabeza de las villas y ciudades del archipiélago. No menos desdeñable era lo que suponía en el componente urbano, al contar con edificios tan emblemáticos como la catedral y el palacio episcopal. Como continuidad histórica, la diócesis trasladada a la ciudad grancanaria será Canariense y Rubicense ${ }^{11}$.

\section{LAS PALMAS: CIUDAD REAL Y EPISCOPAL}

Las Palmas se consolida como cabecera de Gran Canaria y del archipiélago al convertirse en la primera "ciudad real», en territorio administrado directamente por la corona, sin intermediarios de señorío, lo que le dará ventaja sobre las otras islas con su fuero y demás prerrogativas incluidos los símbolos ${ }^{12}$. Contará con toda la cobertura y solidez para el desarrollo de una urbe con un conjunto de circunstancias positivas que hasta el momento no se habían producido en Canarias. El campamento militar, exponente de la estrategia de la conquista, dio paso a un pequeño núcleo civil desde el que se reorganizó Gran Canaria tras la incorporación de 1483, aunque con la particularidad de la permanencia de las dos cabeceras prehispánicas, Telde y Gáldar, que serán las rectoras comarcales del sur y del norte, contando con las primeras parroquias para sendas zonas ${ }^{13}$. Por finalizar la conquista un 29 de abril, la isla se puso bajo el patronazgo de san Pedro de Verona o san Pedro mártir, que precisamente contó con un recinto de culto de su titularidad en cada uno de esos tres lugares: la nueva capital y los dos núcleos canarios antiguos ${ }^{14}$.

Todo el escenario isleño del momento era perfecto para el traslado de la sede del obispado desde Lanzarote a Gran Canaria y Las Palmas estaba preparada. La catedral como elemento más visible del carácter episcopal sería el primer hito de la incipiente urbe y así aparecerá en la pequeña villa que surge en el sitio del campamento junto al lado sur de la desembocadura del barranco de Guiniguada. Era una ciudad con muchos de sus componentes, pero a pequeña escala y con los espacios en relación con su dimensión.

El traslado del obispado por fray Juan de Frías suponía la construcción de la catedral y esta a su vez se convertirá en rectora del proceso arquitectónico y urbano al protagonizar la trama en el área fundacional y en las distintas fases de crecimiento de la población. Un «obispado nómada» que por fin encuentra su sede,

11 Cazorla León: Historia de la Catedral de Canarias, p. 26.

${ }^{12}$ López García, Juan Sebastián: Centros históricos de Canarias, Las Palmas de Gran Canaria, 2011, pp. 111-112. Fuero (1494), escudo de armas (1506), título de ciudad (1515), Real Audiencia (1526), ordenanzas del cabildo (1531), etc.

13 Telde fue sede del primer obispado creado en el archipiélago y Gáldar tuvo la estancia episcopal el obispo Frías mientras terminaba la contienda: Millares Torres, Agustín: Historia General de las Islas Canarias, Las Palmas de Gran Canaria, tomo II, 1975-1981, p. 189.

14 Vid. López García: «Cabeceras históricas y hospitales de Gran Canaria», Revista Aguayro, n. 210 (1994), pp. 17-18. 
un campamento que se convierte en ciudad, mestizaje de personas y un prelado defensor de los naturales, pervivencia de núcleos superpuestos prehispánicos, etc., son factores que convierten a Gran Canaria en una avanzadilla de aspectos que serán poco después muy comunes en América.

\section{LA PRIMERA CATEDRAL: ENCLAVE DE SAN ANTÓN}

La estructura urbana del núcleo fundacional se conoce por el plano de Torriani, realizado un siglo después de la fundación, y porque se ha conservado el trazado. Al ser una zona que quedó anexa al primer ensanche no fue objeto de grandes reformas de alineaciones posteriores, a excepción de la lógica sustitución arquitectónica, ya que poco queda de las primeras construcciones del último tercio del siglo Xv y centuria siguiente ${ }^{15}$.

Se puede considerar que la primera catedral grancanaria es la rectora del núcleo fundacional de la ciudad, dado que la trama urbana presenta una centralidad muy clara, formada por una pequeña plaza (actualmente con el nombre de San Antón o San Antonio Abad [fig. 1]) en la que se produce la convergencia de cinco calles, que de forma estrellada se abren en tramos muy cortos a todos los puntos cardinales, hacia la costa, el barranco y los terrenos colindantes del sur y el oeste. El área pública central se produce por la confluencia de estas vías a lo que se suma un reducido vacío delante de la sede, formando todo la plaza «mayor» que destaca en el escueto perímetro de la villa. La trama es irregular, tanto para la plaza como para el resto del trazado ${ }^{16}$, que se prolonga morfológicamente en el otro lado del barranco de Guiniguada y en forma de vértice confluye en la parte de Triana, donde estaba la ermita de Nuestra Señora de los Remedios (fundada en 1499 y actualmente desaparecida $)^{17}$. Esa zona presenta un escaso número de edificaciones y sólo destaca la referida capilla y su plaza, con una planta que recuerda bastante al «largo portugués», en el sentido de estar conformada por vacíos alrededor del edificio, forma que se ve en otros espacios urbanos canarios. En conjunto, el perímetro total de esta primera fase de Las Palmas tiene una forma triangular, que en esquema ofrece un lado más corto en el sur y dos más largos con punta hacia el norte, con ejes de salidas que conectan con los caminos reales de Telde, Gáldar y La Vega hacia el

15 Tous Meliá, Juan (comisario): Las Palmas de Gran Canaria a través de la cartografía (1588-1899), Las Palmas de Gran Canaria, 1995, pp. 35-41.

16 Vid. Fraga González, Carmen: «Plazas de Las Palmas». En III Coloquio de Historia Canario-Americana, Las Palmas de Gran Canaria, tomo II, 1980, pp. 299-300.

17 Vid. Cazorla León, Santiago: «La ermita de los Remedios de Las Palmas», en Homenaje a D. Agustín Millares Carló, Las Palmas de Gran Canaria, tomo II, 1975, pp. 225-253. Herrera Piqué, Alfredo: La ciudad de Las Palmas. Noticia histórica de su urbanización, Las Palmas de Gran Canaria, 1978, pp. 45-46. 


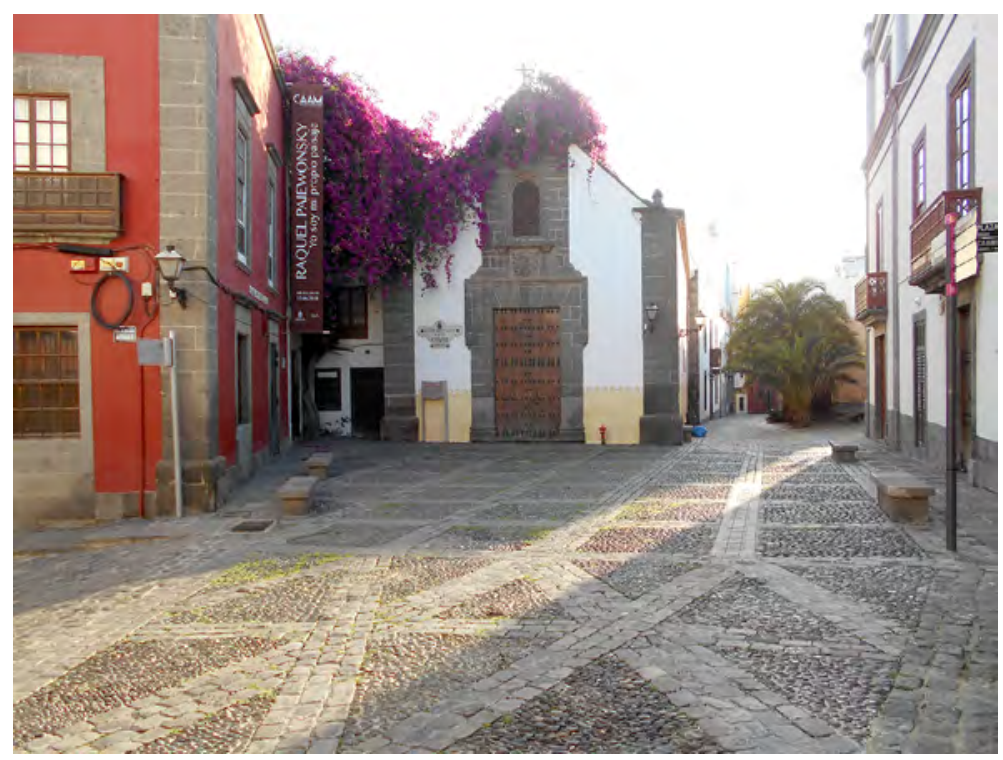

Fig. 1. Plaza de San Antonio Abad, Las Palmas de Gran Canaria (marzo de 2018).

interior de la isla, mientras la cercana Caleta de San Telmo, puerto de las Isletas y otros fondeaderos comunican por mar con el exterior.

Muy modestos alardes constructivos se suponen en los años siguientes a la conquista, cuando aún faltaba por integrar a las islas de La Palma y Tenerife, en los años 1493 y 1496. Desde el punto de vista arquitectónico poco se puede decir, más allá de la «huella» del solar que hoy ocupa la ermita de San Antonio Abad, cuya dimensión revela la modestia de la primera sede catedralicia grancanaria, aunque posiblemente fuera la de mayor entidad de las existentes hasta ese momento en relación con los tres antecedentes de Telde, Rubicón y Betancuria. Es probable que no se distanciara mucho de las iglesias parroquiales que casi a la par se levantaban, en esa urgencia de crear la infraestructura religiosa que necesitaba la isla.

\section{LA IGLESIA VIEJA}

La inmediatez con que se construiría el primer recinto cated ralicio, en pleno período de posconquista, los cambios que se producen con la integración de La Palma y Tenerife, el desarrollo económico de la isla y el crecimiento de la pequeña ciudad son elementos que pudieron incidir en la construcción casi a la par de dos nuevas construcciones de características diferentes. La más antigua parece ser la «iglesia vieja», de la que no se conoce la fecha del inicio de las obras y que según Cazorla León no se construyó en la época del obispo Juan de Frías, sino que fue más tardía, con un período constructivo en torno a 1514, aunque finalizada y abierta al culto 


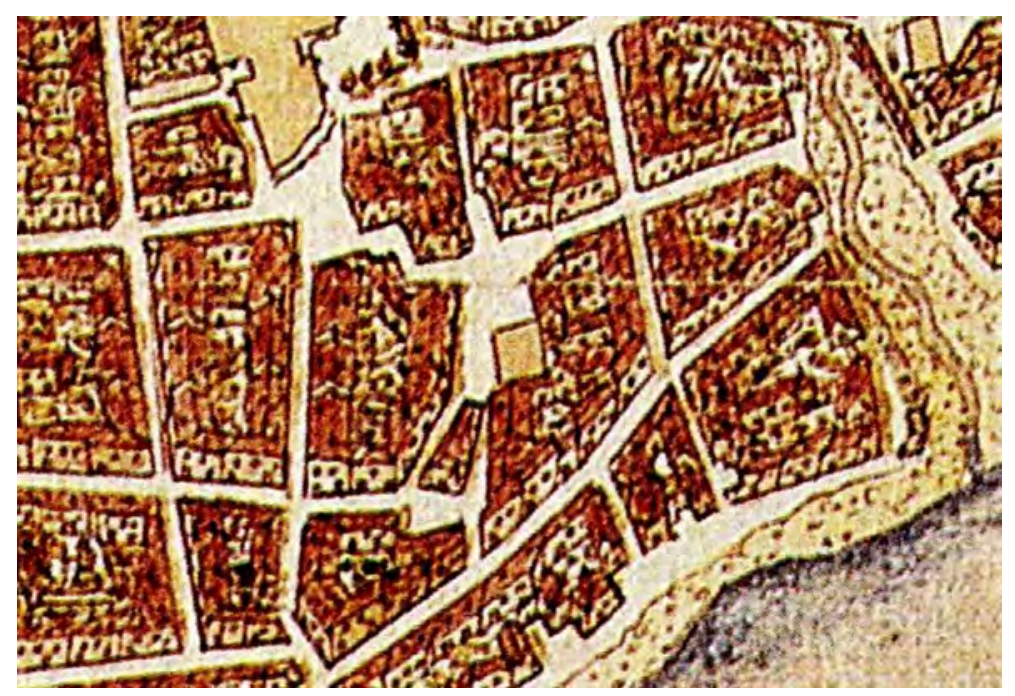

Fig. 2. Plaza de San Antonio Abad, Las Palmas de Gran Canaria.

Plano de Leonardo Torriani (ca. 1588).

en menos de dos décadas. Se la denominaba «iglesia vieja» en 1536, aunque también se la conocerá como «iglesia baja», ambos nombres en relación con la fábrica de la catedral propiamente dicha. Era pequeña, ya que en 1551 se afirma: «El coro y el altar y todo el servicio de la dicha iglesia no podrá haber más de hasta treinta pasos en largo y quince en ancho, y antes menos que más, y lo que queda para donde la gente se recoge a oír los divinos oficios habrá la distancia doblada» ${ }^{18}$.

Colmatado el primer núcleo y analizando la trama urbana, se aprecia que se acudió al borde del lado oeste, donde están otras dos plazuelas, aún con las mismas características que la de San Antón, pero sin su rasgo de centralidad, ya que ocupan posición en el borde de la primitiva villa. En el plano de Torriani (ca. 1588) [fig. 2] y la cartografía hasta el siglo XviII se mantiene el trazado e imagen que la plaza de los Álamos o de las Gradas debió tener a finales del siglo xv, donde estaba la escalinata de la iglesia vieja, y de ahí uno de los nombres del enclave ${ }^{19}$, con la casa del gobernador y el hospital de San Martín ${ }^{20}$. Quedaba muy cerca de la anterior catedral y

18 Cazorla León, op. cit., pp. 455-463. Hernández Gutiérrez, A. Sebastián (coordinador): La Catedral de Santa Ana. Patrimonio Histórico. Las Palmas de Gran Canaria, Santa Cruz de Tenerife, 1999, pp. 20-22.

19 Tous Meliá (comisario): op. cit, pp. 41, 50, 54, 71, 78, 84, 102 y 105.

20 Vid. Bosch Millares, Juan: El Hospital de San Martín. Estudio histórico desde su fundación hasta nuestros días, Las Palmas de Gran Canaria, 1940. 
sólo había que recorrer la actual calle Colón para ir a sus correspondientes plazuelas. Su espacio público sigue comunicado con el resto de la villa y del territorio de una manera similar a la primera plaza, con una calle que baja hacia el barranco para conectar con el lado de Triana y enlazada con la otra plazuela, conocida desde el siglo XviII por el nombre del Pilar Nuevo, que al parecer de Cazorla estuvo en sus orígenes unificada espacialmente a su colindante de los Álamos.

Este edificio debió cumplir los cometidos de primer templo de Canarias hasta el traslado de la sede a la "media catedral», construcción a la que estaba adosado. La documentación aportada por Cazorla León relativa a este edificio parece confirmar que cumplió funciones catedralicias, lo que posiblemente sucedió hasta la apertura al culto de la fábrica gótica. En 1551 se refiere lo siguiente: «el cuerpo de la iglesia (baja), donde Señores Deán y Cabildo celebran el culto y oficios divinos es muy estrecho y pequeño, y tanto que en él cabe muy poca gente, como cada día se ve», lo que confirma la presencia cotidiana de las dignidades propias de la catedral como si fuera edificio propio ${ }^{21}$. Sin embargo, la principal función del inmueble fue la de parroquia del Sagrario, que tenía anexos varios curatos de la isla: inicialmente Arucas, Moya, La Vega, Tirajana y Teror, con la particularidad de Agüimes (cámara episcopal) y de los integrados en los beneficios independientes de San Juan de Telde y Santiago de Gáldar ${ }^{22}$.

Fue ampliado en el siglo xvir cuando ejercía exclusivamente de parroquial, ya que el cabildo de 2 de julio de 1629 acuerda «se haga conforme las tres naves del cuerpo de la iglesia de Santo Domingo». Llegó a tener tres naves y siete capillas, la mayor y tres a cada lado del inmueble (Bautisterio o San Juan Bautista, Virgen de Belén, San Andrés, San Miguel, Virgen de la O y Virgen de la Antigua) y se mantuvo hasta $1780^{23}$. Por los planos de la ciudad de los siglos XVI al XVIII se aprecia que su tamaño era notablemente superior a la anterior del recinto de San Antón, prácticamente el doble, con una cierta irregularidad en su planta.

\section{LA MEDIA CATEDRAL Y LA PLAZA MAYOR}

Como se ha visto, a menos de veinte años de conquistada la isla, la ciudad siguió evolucionando y hacia 1500 se plantea una nueva catedral, con un proyecto ambicioso y de futuro, el cual no pudo ser asumido en su conjunto y se acometió parcialmente en una primera fase. Durante el siglo Xvi se construirá la «media catedral» con obras que se dieron por finalizadas en el último tercio. Es con esta fábrica cuando se tienen más datos y nombres de los artífices, aparte de ser una obra que se conserva en buena parte, especialmente en lo que respecta al interior, ya que la desaparecida fachada sólo se conoce por dibujos, no demasiado exactos.

21 Cazorla León, op. cit., p. 461, véanse también los ítems viII, IX y XII.

22 Cazorla León, op. cit., pp. 11-24.

23 Cazorla León, op. cit., pp. 18, 459, 466-510. 
Los inmuebles anteriores relacionados con el culto diocesano se levantaron en los espacios destacados de cada momento en un núcleo unitario aunque muy pequeño. En esta tercera ocasión será con más claridad el exponente del crecimiento que se había operado en la ciudad y precisamente la nueva y definitiva catedral irá unida a un programa urbano que supondrá el primer ensanche de lo que había sido el núcleo fundacional. Esta sede catedralicia se concibe en la nueva plaza mayor, de planificación renacentista, lugar hacia donde recaerá su fachada principal, siendo el espacio en el que se concentrarán durante el siglo Xvi y primeras décadas del XVII los edificios institucionales más importantes de Canarias y, por ende, de Gran Canaria: la catedral, el obispado y la Real Audiencia, todos ellos con ámbito en el archipiélago, y el Cabildo que era el gobierno de la ciudad y de la isla.

Este edificio adquiere una dimensión urbana que no había tenido ninguno de los anteriores por su ubicación en el amplio rectángulo que es la nueva y definitiva plaza mayor, de regularidad renacentista y que se puede considerar un precedente para las americanas, con las que coincide si no tanto por su morfología sí por su multifuncionalidad y concentración de los poderes ${ }^{24}$.

La fábrica se inicia bajo los postulados del gótico, que todavía regían en Espańa y Portugal, donde era muy fuerte la sugestión de las catedrales en este estilo, en especial la de Sevilla para el ámbito canario. Los cimientos se abren en tiempos del obispo Diego de Muros y el primer artífice fue Diego Alonso de Montaude, quien trabaja hasta 1504, año en que se encarga de las mismas Pedro de Llerena (1504-1519). Entre 1519 y 1533 la dirección quedó vacante, hasta que se hizo cargo de ellas Juan de Palacios (1533-1551), que trabaja en las célebres columnas anilladas, la fachada y las torres, sucediéndole Martín de Barea o Narea (1553-1561) con sus intervenciones en las capillas laterales y bóvedas de nervadura, con la posibilidad de haber ideado la «Puerta del Aire», con trazado renacentista, dentro de la tendencia de consolidación de este estilo en Canarias. Su sobrino, Pedro Narea (1562-1585), incorporaría también las formas clásicas y tendrá el honor de cerrar la catedral en 1570. En los ańos posteriores se suman algunas intervenciones, como la «puerta mayor», trazada por el italiano Próspero Cassola, también renacentista. Poco antes de finalizar el siglo Xvi se daba por terminada la «media catedral», quedando por concluir algunas capillas. La obra había comenzado por los pies (fachada hacia la plaza

24 AA. VV.: La Plaza Mayor de Las Palmas de Gran Canaria y las Plazas Mayores Iberoamericanas, Las Palmas de Gran Canaria, 2010. López García, Juan Sebastián: «La Plaza Mayor en Canarias como espacio del poder", en Documentos de Arquitectura Nacional y Americana (DANA), n. ${ }^{\circ}$ 31/32, (1992), pp. 108-112. López García, Juan Sebastián: «La Plaza del Pilar Nuevo (Las Palmas de Gran Canaria)", en San Juan 93. Textos de las Fiestas Fundacionales. 1478-1993. Las Palmas de Gran Canaria, 1994, pp. 197-203. López García, Juan Sebastián: «La Plaza de Santa Ana de Las Palmas de Gran Canaria. El espacio y la imagen polifacéticos», en La Plaza de Santa Ana y su entorno, Las Palmas de Gran Canaria, 2001. pp. 41-53. López GarCía, Juan Sebastián: «Las plazas, un espacio público concomitante panhispánico», en Patrimonio cultural: intertextos y paralelismos, Zapopan (México), 2003, pp. 113-134. 
de Santa Ana) y sólo se trabajó hasta la mitad de sus naves, en el solar hacia la futura cabecera. La otra «media catedral» tuvo que esperar poco más de doscientos ańos ${ }^{25}$.

El rápido repaso de autores y cronologías, sin entrar en ninguno de sus aspectos, pone en evidencia la complejidad de la obra en una ciudad pequeńa y en un obispado que prácticamente iba arrancando, con un frente abierto de programas constructivos de parroquiales prácticamente en todas las islas, dándose el caso de que algunos de los artífices que trabajan en Santa Ana también lo hacen en templos de Gran Canaria o de otras islas como La Palma, donde Juan de Palacios aparece trabajando en la iglesia de El Salvador (Santa Cruz de La Palma) ${ }^{26}$. De cualquier manera, ningún edificio canario le era semejante, sobre todo por su tamaño y la gran sala de naves abovedadas, que contrastaba con las cubiertas de madera de tradición mudéjar que predominaban en el resto de los edificios religiosos isleños.

En realidad el hecho de sólo haberse construido la media catedral no afectaba demasiado a su dimensión urbana: su fachada principal estaba ubicada y presidía ese gran «salón urbano» que es la plaza mayor a la que da nombre Santa Ana, frente al Cabildo de Canaria, concejo de la isla. Esa imagen del edificio será hegemónica en Canarias durante casi dos siglos, donde tendrá la exclusiva de tener una fachada con dos torres gemelas, ya que ninguna de las iglesias parroquiales de las islas contaba con esa característica y las principales, incluso las conventuales que dispusieron de recursos, eran de torre única. Algunas de esas torres eran notables como las de El Salvador de Santa Cruz de La Palma y las correspondientes a la Concepción lagunera y a la santacrucera. La arquitectura de Santa Ana, tanto en el interior como en el exterior, era la «imagen catedralicia» de Canarias y así influyó en otros edificios que repitiendo de forma anacrónica el lenguaje artístico de la misma adquirían el prestigio de una marca reconocida en las islas, por eso no es de extrañar que en Teror se imitara en 1708 una de las torres de la fachada de Santa Ana ${ }^{27}$, y que la capilla mayor de la iglesia de Nuestra Señora de la Concepción en San Cristóbal de La Laguna (posiblemente a partir de 1785) imitara baquetones y cubiertas de crucería de la sede canariense ${ }^{28}$. En ambos casos fueron recurrentes en cada uno de sus ámbitos, en Teror en un momento de auge de la devoción a Nuestra Señora del Pino, consolidando su santuario en el ámbito grancanario, mientras en La Laguna, aparte de las obras que precisaba el templo concepcionista, es muy posible que el lenguaje artístico formara parte de la tradicional rivalidad entre la parroquia matriz

25 Hernández Perera, Jesús: Los arquitectos de la Catedral de Las Palmas, Las Palmas de Gran Canaria, 1998, pp. 43-133. Cazorla León: op, cit., pp. 47-65. Hernández Gutiérrez (coord.): op. cit., pp. 22-32 y 48-49. Galante Gómez, Francisco: Elementos del gótico en la arquitectura canaria, Las Palmas de Gran Canaria, 1983, pp. 83-91. López García, Juan Sebastián: Arquitectura del Renacimiento en el Archipiélago Canario, La Laguna, 1983, pp. 145-150.

${ }^{26}$ Rodríguez, Gloria: La iglesia de El Salvador de Santa Cruz de La Palma, 1985, Madrid, p. 11.

27 Vid. Quintana, Ignacio y Cazorla, Santiago: Nuestra Señora del Pino de la Historia de Gran Canaria, Las Palmas de Gran Canaria, 1971, pp. 175-183. Sánchez Rodríguez, Julio: Las iglesias de Nuestra Señora del Pino y las ermitas de Teror, Santa Cruz de Tenerife, 2008, pp. 203-209.

${ }_{28}$ Lorenzo Lima, Juan Alejandro (ed.) (2016): La Laguna y su parroquia matriz. Estudios sobre la Iglesia de la Concepción, Instituto de Estudios Canarios, La Laguna, 2016, pp. 116-139. 


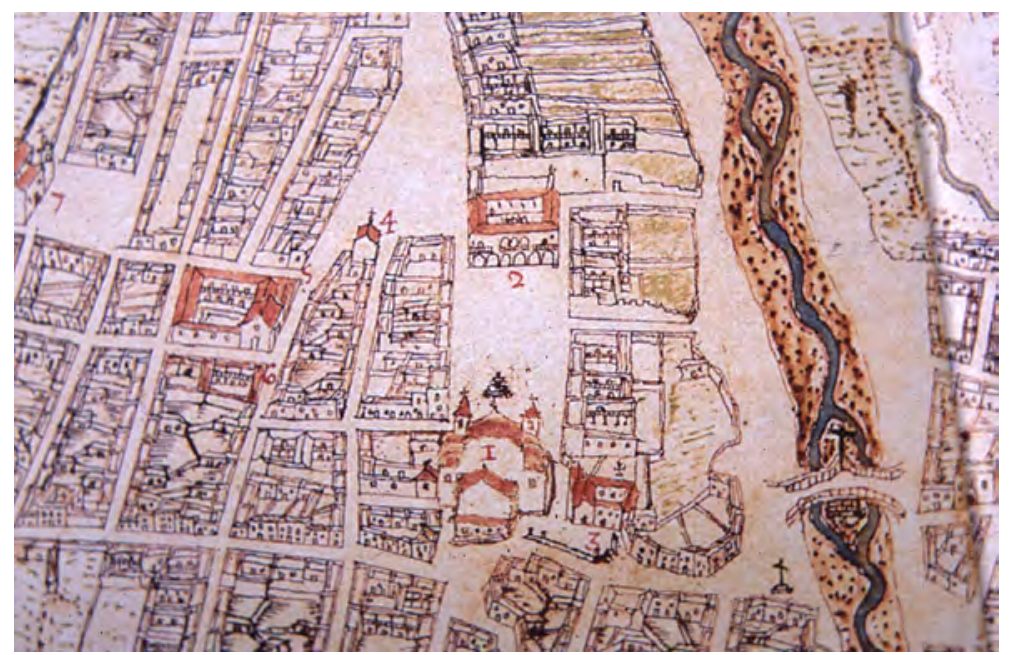

Fig. 3. Plaza de Santa Ana, Las Palmas de Gran Canaria. Plano de Pedro Agustín del Castillo (1686).

y la de Nuestra Señora de los Remedios, reforzando el aspecto de la primera sobre la segunda.

La catedral de Santa Ana mantuvo desde el siglo XvI, durante el siglo XVII y parte del XVIII su imagen hegemónica de fachada monumental en Canarias, protagonizando la plaza mayor de Las Palmas, el espacio público más significativo del archipiélago, como «plaza de los poderes». Esta situación se modifica en el siglo XviII, con algunas aportaciones arquitectónicas [fig. 3], donde van a destacar dos iglesias parroquiales por sus imafrontes monumentales: Nuestra Señora de la Concepción, de tres naves con capillas hornacina, cubierta con bóvedas y cúpula sobre pechina, artística fachada barroca y torres pareadas (La Orotava, 1768), y Santiago de los Caballeros, neoclásica, de tres naves con quince capillas, cubierta de bóvedas con cúpula sobre pechinas, fachada totalmente en cantería y torres gemelas (Gáldar, $1778)^{29}$. Junto a estos inmuebles hay otros de menor notoriedad, siendo en este el contexto en el que en 1781 se reanudan las obras de la catedral de Santa Ana.

29 Vid. López García, Juan Sebastián: «Promoción, mecenazgo y cambio en la arquitectura religiosa de Gran Canaria, siglo XviII", en VII CEHA. Patronos, Promotores, Mecenas y Clientes, CEHA, Murcia, 1992, pp. 519-530. CALERo Ruiz, Clementina et alii: Luces y sombras en el siglo ilustrado. La cultura canaria del Setecientos, Santa Cruz de Tenerife, 2008, pp. 62-63. 


\section{LA CULMINACIÓN DE UN PROCESO ABIERTO: LA CATEDRAL, SIGLOS XVIII-XX}

Después de la paralización de las obras en el último tercio del siglo xvi y superado el debate del estilo a utilizar en la continuación de la catedral, en 1781 se reanudan las obras bajo la dirección de Diego Nicolás Eduardo Villarreal (17331798), quien mantiene la unidad estilística del gótico en el interior e introduce el neoclásico para los exteriores. El bello proyecto eduardiano supone la culminación de la otra "media catedral» hacia la cabecera, incluyendo la propuesta de construir la iglesia del Sagrario en el lado norte. A su muerte, José Luján Pérez (Santa María de Guía, 1756-1815) continúa al frente de la fábrica, firmando en 1809 el proyecto de fachada, que integraba también la colindante parroquia del Sagrario ${ }^{30}$. Culminado el interior del templo, el principal empeño fue la fachada principal, para la que se realizaron diversas propuestas durante el siglo XIX, completando o modificando las trazas lujanianas: E. Demangeat (1848), M. Oráa (1854), F. Jareńo (1860), J. de la Gándara (1869), V. Mananzoni (1895), A. Mélida (1896), Laureano Arroyo (diseńo de 1898) y finalmente, siguiendo las directrices del proyecto del último arquitecto, Fernando Navarro la culmina en $1901^{31}$.

El proyecto en planta de Eduardo y el alzado de fachada de Luján se complementan y explican perfectamente la propuesta de culminación del conjunto, que no se completó al no culminarse la iglesia del Sagrario. La obra no es sólo importante y significativa en cuanto a lo arquitectónico, sino también en lo urbano. El proyecto de Diego Nicolás (1784) suponía también la primera reforma anexa de la plaza de Santa Ana, ya que con la construcción de la nueva parroquia del Sagrado al lado norte de la catedral (planteada sobre la calle inmediata y el hospital de San Martín) se proyectó delante de la misma la «Plazuela destinada en este Proyecto a la nueva Parroquia», que constituía un apéndice de la «Plaza mayor de Santa Ana», quedando formada entre la esquina del palacio episcopal, cerrada en su fondo y con una calle de salida hacia la zona de San Antón por la «calle nueva del Sagrario» (actual San Marcial). En la cabecera del conjunto catedralicio, la fábrica modificaba la planta de las antiguas plazuelas de San Martín (las Gradas o los Álamos) y del Pilar Nuevo ${ }^{32}$. Años después, en el siglo XIX, se complementó la operación urbana con la apertura de una calle (calle Nueva, actual Obispo Codina, más estrecha en origen), que partiendo del fondo de la plazuela del Sagrario va a comunicar directamente la plaza de Santa Ana con el barrio de Triana, salvando el barranco de Guiniguada por el "puente de piedra», regalo del obispo Verdugo y diseñado por Luján Pérez en 1815. Con esta intervención la plaza mayor y la catedral de Santa Ana quedarán perfectamente

${ }^{30}$ Galante Gómez, Francisco: El ideal clásico. Arquitectura canaria (desde la segunda mitad del siglo XVIII hasta finales del siglo XIX), Las Palmas de Gran Canaria, 1989, pp. 122-128. Cazorla LeÓN, op. cit, pp. 65-73, 74-75. Hernández Gutiérrez (coord.): op. cit., 50-52.

31 Hernández Gutiérrez (coord.): op. cit., pp. 42-46, 131-135. Galante Gómez: op. cit., pp. 128-134.

32 Vid. plano en Tous Melía (comisario): op. cit., pp. 92-99. 
comunicadas con el resto de la ciudad, en una iniciativa que marcará el inicio de las transformaciones urbanas decimonónicas que afectarán a ambos barrios, Vegueta y Triana, pero con mayor incidencia en el trazado del segundo, teniendo su principal aporte en la alameda de San Francisco y plaza de Cairasco ${ }^{33}$. Al mismo tiempo, la catedral refuerza su presencia en Canarias al dotarse de la fachada más monumental del archipiélago y la plaza de Santa Ana asistirá a lo largo del siglo XIX a una notoria modificación de imagen, no sólo por el frontis catedralicio, sino por la construcción de las nuevas casas consistoriales y la segunda planta de la Casa Regental. El cambio de aspecto y «modernización» de la plaza mayor no era una cuestión secundaria en el momento político que se vivía, ya que Las Palmas era candidata a cabecera de Canarias y ese conjunto urbano y arquitectónico se ofrecía como un buen espacio público para una capital provincial, lo que reforzaba a Las Palmas en sus aspiraciones en la escena regional, especialmente en cuanto al pleito insular.

En el ámbito territorial eclesiástico, el acontecimiento más importante del siglo XIX en el archipiélago fue la creación del obispado de San Cristóbal de La Laguna, con jurisdicción en las Canarias occidentales, lo que supondrá una segunda catedral en las islas, con sede en la lagunera iglesia Nuestra Señora de los Remedios ${ }^{34}$.

\section{CONCLUSIONES}

Las diferentes catedrales que ha tenido el archipiélago canario evidencian en gran medida el devenir histórico de las islas y la expansión europea por el Atlántico medio. Sus localizaciones en el territorio respondieron a estrategias de distinto tipo en relación con la geopolítica del momento, antes y después de la conquista castellana, en islas de señorío e islas de realengo. El traslado de la diócesis de Rubicón a Las Palmas constituyó la consolidación del obispado y el inicio de un proceso constructivo que desde 1483 llegó hasta principios del siglo Xx.

La catedral de Santa Ana [fig. 4] fue la iniciativa constructiva más importante y significativa en la historia de las islas. Los distintos edificios que han sido su sede han formado parte directa del proceso urbano de la capital grancanaria y los espacios públicos que en cada momento histórico han constituido su centralidad principal. Fue un edificio hegemónico e influyente en Canarias que marcó pautas constructivas en otros inmuebles del territorio isleño y que transmitía una imagen positiva de Las Palmas más allá de lo religioso, con trascendencia en las reivindicaciones

${ }^{33}$ López García, Juan Sebastián: «La ciudad y el urbanismo del siglo XIx en Canarias», en Un artista para una ciudad y una época. Manuel Ponce de León, Las Palmas de Gran Canaria, tomo I, 2004, p. 22.

${ }^{34}$ La bula de erección del obispado de San Cristóbal de La Laguna por el papa Pío VII es del 1 de febrero de 1819, suspendido en 1851 y gobernado por el obispo de Canarias como Administrador apostólico, fue restablecido en 1877 (SÁNCHez Rodríguez, La Iglesia en las Islas Canarias, p. 137). Vid. Núñez Muñoz, María F.: La Diócesis de Tenerife. Apuntes para su historia (1815-1899), Santa Cruz de Tenerife, 1986. 


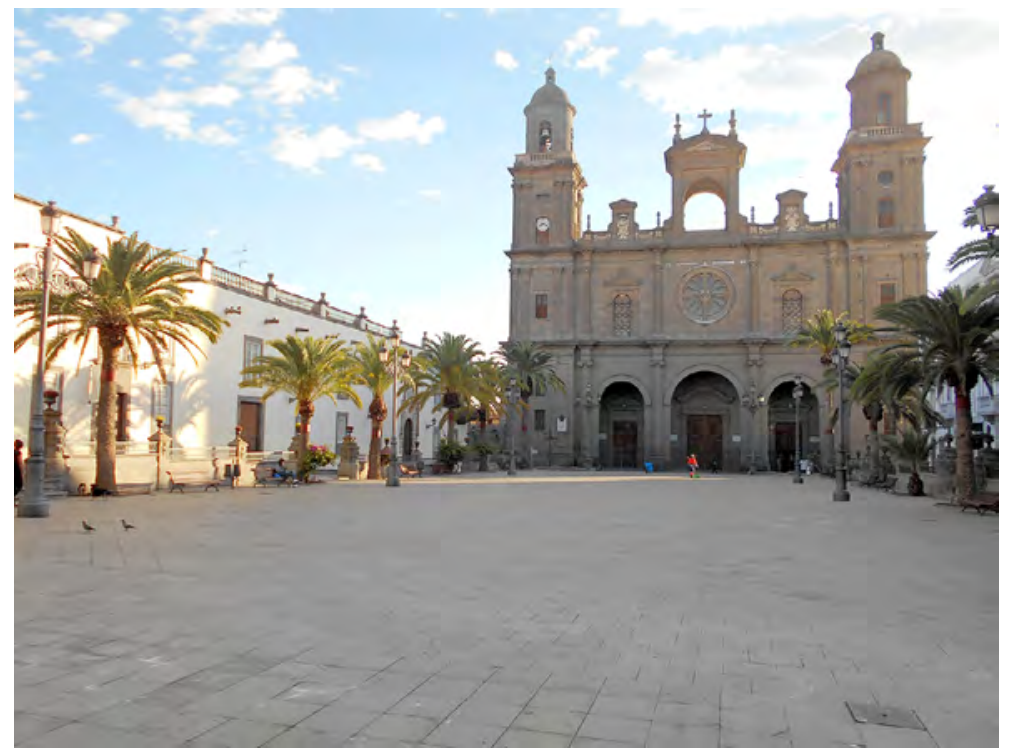

Fig. 4. Plaza de Santa Ana, Las Palmas de Gran Canaria (marzo de 2018).

políticas de la ciudad. Catedral/plaza mayor ha sido un binomio arquitectónico y urbano como principal escenario de centralidad y espacio público que concentró los poderes institucionales más importantes del archipiélago. "Monumento insigne del patrimonio artístico canario», como la calificó Jesús Hernández Perera.

Recibido: 21-03-2018, ACEPTADO: 10-04-2018 
